\title{
Hypertrophic pulmonary osteoarthropathy with esophageal sarcomatoid carcinoma: a case report
}

\author{
Maoyuan Zhao ${ }^{1}$, Yanru Du' ${ }^{2}$, Qing Peng ${ }^{3}$, Zhouguang Hui ${ }^{1}$ \\ ${ }^{1}$ Department of Radiation Oncology, Department of VIP Medical Services, National Carcinoma Center/National Clinical Research Center \\ for Carcinoma/Carcinoma Hospital, Chinese Academy of Medical Sciences \& Peking Union Medical College, Beijing, China; ${ }^{2}$ Department of \\ Pathology, National Carcinoma Center/National Clinical Research Center for Carcinoma/Carcinoma Hospital, Chinese Academy of Medical \\ Sciences \& Peking Union Medical College, Beijing, China; ${ }^{3}$ Nuclear Medicine Department, National Carcinoma Center/National Clinical Research \\ Center for Carcinoma/Carcinoma Hospital, Chinese Academy of Medical Sciences \& Peking Union Medical College, Beijing, China \\ Correspondence to: Zhouguang Hui. Department of Radiation Oncology, Department of VIP Medical Services, National Carcinoma Center/National \\ Clinical Research Center for Carcinoma/Carcinoma Hospital, Chinese Academy of Medical Sciences \& Peking Union Medical College, Panjiayuan \\ Nanli \#17, Chaoyang District, Beijing 100021, China. Email: huizhouguang@yeah.net.
}

\begin{abstract}
Hypertrophic pulmonary osteoarthropathy (HPOA), mainly manifested clubbing, is rare in patients with esophageal sarcomatoid carcinoma. We herein describe a 48-year-old Chinese man whose advanced sarcomatoid carcinoma was diagnosed while examining his symptoms of HPOA. The patient had no opportunity of surgery after surgical evaluation. Chemoradiation, including 5 cycles of chemotherapy (Paclitaxel liposome $60 \mathrm{mg}$ day 1 and nedaplatin $30 \mathrm{mg}$ day 1, q1w) and 6MV-X/VMAT-95\%PGTV 59.92 Gy/2.14 Gy/28 F and 95\%PTV 50.4 Gy/1.8 Gy/28 F, and subsequent 6 cycles of chemotherapy (paclitaxel liposome $210 \mathrm{mg}$ day 1 and nedaplatin $50 \mathrm{mg}$ day 1, $60 \mathrm{mg}$ day 2, q3w) shrank the tumor and the condition of the patient became stable without clubbing remission or exacerbation. No medical case report found in a PubMed search in the indexed English-language literature in the past 20 years, though there are some reports of HPOA combined with other pathologic types of esophageal carcinoma. The patient's condition was effectively controlled by chemotherapy and radiotherapy and showed stable disease. However, the five-year survival rate of advanced esophageal carcinoma patients is very low with low life quality, and the adverse reactions also contribute to low life quality. The purpose of this report is to present the feature and our treatment for primary esophageal sarcomatoid carcinoma with HPOA, which could be helpful for further understanding of the disease and clinical decision making. Moreover, this article also reviews esophageal carcinoma with HPOA and sarcomatoid carcinoma in the esophagus. We look forward to the breakthrough of immunotherapy and molecular targeting therapy to improve the situation.
\end{abstract}

Keywords: Hypertrophic pulmonary osteoarthropathy (HPOA); esophageal sarcomatoid carcinoma; clubbing; case report

Submitted Jun 25, 2020. Accepted for publication Aug 28, 2020.

doi: 10.21037/apm-20-1309

View this article at: http://dx.doi.org/10.21037/apm-20-1309

\section{Introduction}

Hypertrophic osteoarthropathy (HOA) is a syndrome caused by thickening of soft tissue around the bone and extensive periosteal new bone formation, its age of onset is mainly middle-aged and old people (1). The main clinical manifestations are clubbing fingers (toes) and extensive periosteal hyperplasia of the long bone (1). This disease can be divided into two types-primary HOA that is also called familial HOA and secondary HOA that often has obvious visceral diseases, the most of which is lung, is also known as hypertrophic pulmonary osteoarthropathy (HPOA) (2). Sarcomatoid carcinoma is rare in esophageal carcinoma. Its biological behavior and prognosis are different from other common esophageal carcinoma pathologic types- 
A
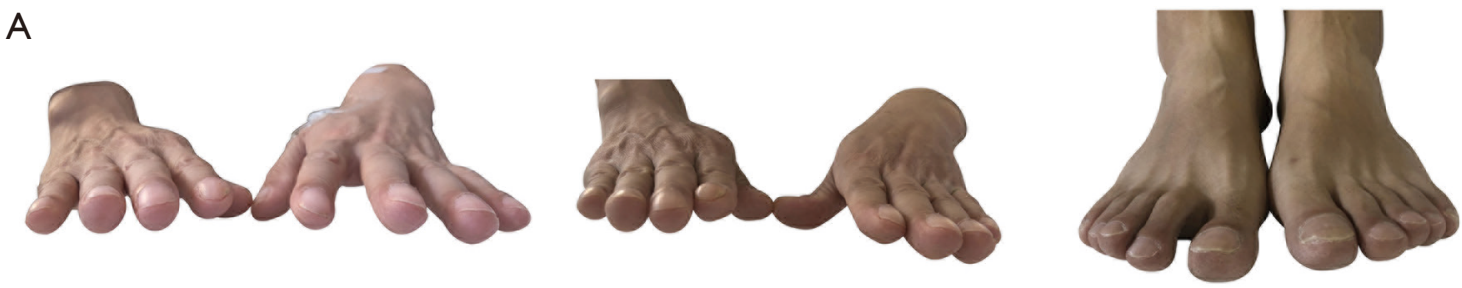

B

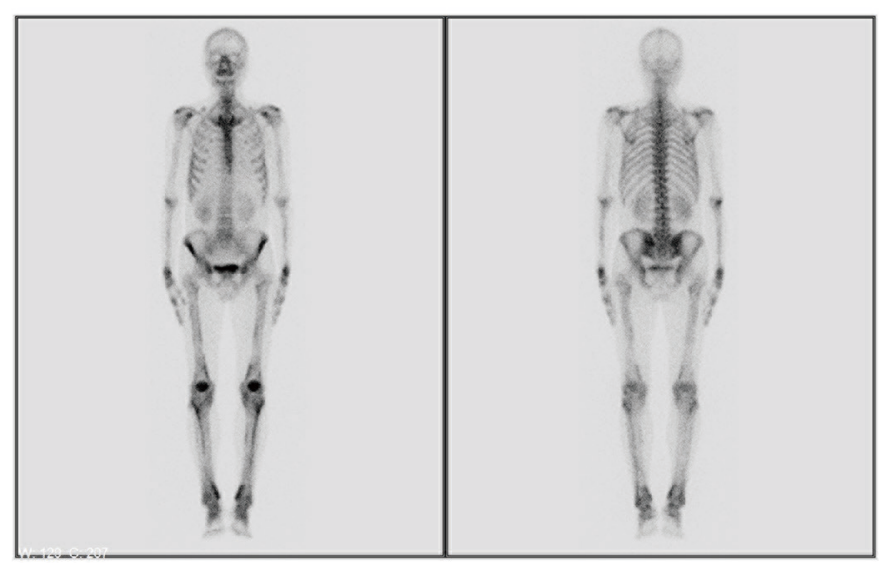

Figure 1 Clinical manifestations and SPECT examination. (A) Clubbing of the fingers and toes. Symmetric clubbing was found in the fingers (left and middle) and toes (right). (B) Technetium-99m labeled MDP (methyl diphosphonate) bone scan of the whole body shows increased uptake at the hands, feet, proximal humerus, proximal femur, and tibia bilaterally.

esophageal squamous carcinoma and adenocarcinoma $(3,4)$. So HPOA in esophageal sarcomatoid carcinoma is rare. Our study aims to describe and discuss the clinical feature, imaging, pathological features, and treatment of the rare case. We present the following article in accordance with the CARE reporting checklist (available at http://dx.doi. org/10.21037/apm-20-1309).

\section{Case presentation}

A 48-year-old male has difficulty swallowing, choking with food, and chest and back pain in August 2018. No improvement after 1-month reating pharyngitis. At the beginning of September 2018, the fingers and toes began to hypertrophy (Figure 1A), and there was no tenderness or increased skin temperature. Single-photon emission computed tomography (SPECT) bone scan showed increased radioactivity in the long bone cortex and joints of the four limbs (Figure 1B) and it was considered as HPOA. His pulmonary function test revealed a large extrapolated volume, insufficient explosive power, and a platform of the expiratory curve (Figure 2), which conformed to the possibility of intrathoracic or fixed upper airway obstruction.
And he had decreased forced vital capacity (FVC) with normal forced the first second of expiratory volume (FEV1) and normal total lung capacity (TLC), which was consistent with obstructive ventilation dysfunction. Finger oxygen was $95-96 \%$. Before that, Upper digestive tract radiography revealed that the mucosa was damaged about $7.7 \mathrm{~cm}$ in the middle thoracic esophagus, irregular niches were seen, and the local lumen was expanded (Figure $3 A$ ). Computed tomography $(\mathrm{CT})$ revealed solid mass in the root of lower neck (Figure 3B), the side of esophagus, tracheoesophageal groove, $4 \mathrm{~L}$ and 8 section of mediastinal lymph nodes, and the side of cardia, the largest of which invaded the pericardium and the root of pulmonary vein and was $3.8 \times 2.4 \times 6.6 \mathrm{~cm}^{3}$ and, in the middle thoracic esophagus (Figure 3C). Ultrasonography also revealed abnormal cervical lymph nodes with low echo. Endoscopy (Figure $4 A$ ) with biopsy and endoscopic ultrasonography (Figure $4 B$ ) revealed that a protuberant mass, mainly located in the intrinsic myometrium and penetrated the outer membrane esophagus, was about $27-36 \mathrm{~cm}$ from the incisor with unclear boundary with surrounding tissues. In addition, hyperemia and coarseness of the left pyriform fossa were also observed by endoscopy with 


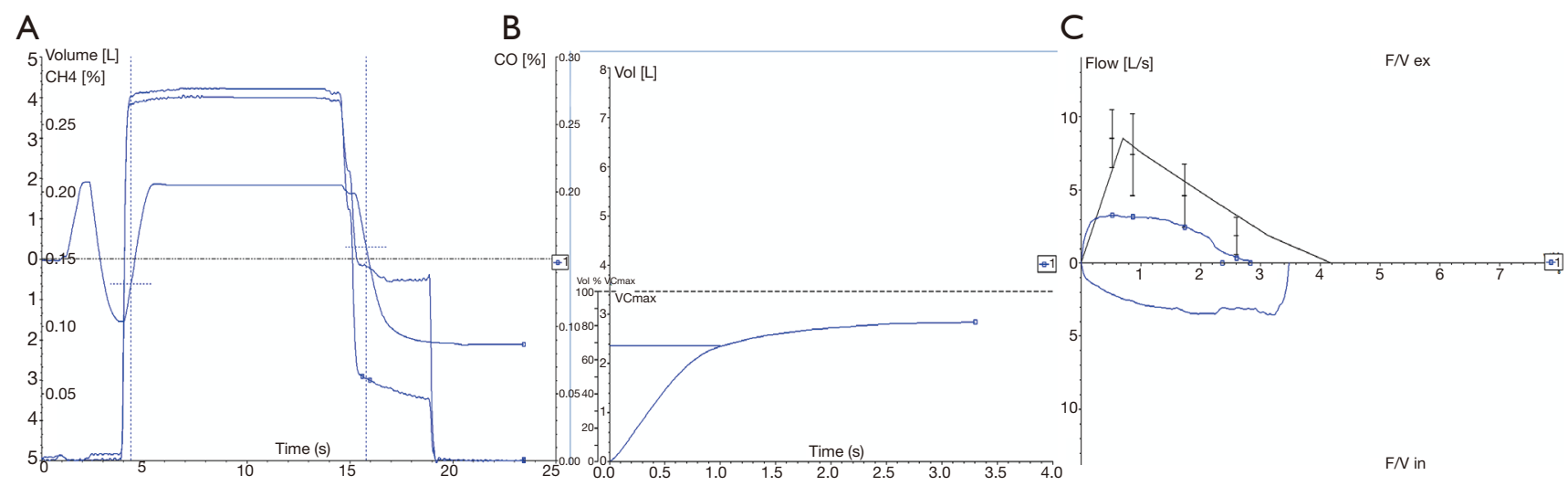

Figure 2 Pulmonary function test. (A) Diffusion function curve. (B) Time volume curve. (C) Flow-volume curve.

biopsy. Hematoxylin and eosin (HE) staining and Vimentin immunohistochemical staining of the esophagus tissue and HE staining of the left pyriform fossa indicated sarcomatoid carcinoma(Figure $5 A, B$ ) and squamous cell carcinoma (Figure 5C), respectively. SPECT bone scan and abdominal CT failed to show evidence of other distant metastasis and special exceptions. No tumor markers were remarkable. The patient's medical history did not show evidence of carcinoma or other disease and any family member had not been diagnosed with carcinoma. This patient has been smoking and drinking for 27 years, with about 20 cigarettes and $300 \mathrm{~g}$ white wine per day, and has now quit smoking and drinking for one month. His Karnofsky Performance Status (KPS) grade was 80 and blood routine and blood biochemical test was normal. Based on the above results, the final diagnosis was esophageal sarcomatoid carcinoma and squamous cell carcinoma of pyriform fossa with HPOA, and the tumor node metastasis (TNM) classification was cT4N1M1b in esophageal sarcomatoid carcinoma and cT1N0M0 in squamous cell carcinoma of pyriform fossa, which corresponds to at least American Joint Committee on Carcinoma (AJCC) Stage IVB and I respectively. The patient received concomitant chemoradiotherapy from October to December 2018, including 5 cycles of chemotherapy (paclitaxel liposome $60 \mathrm{mg}$ day 1 and nedaplatin $30 \mathrm{mg}$ day $1, \mathrm{q} 1 \mathrm{w}$ ) and $6 \mathrm{MV}$-X/VMAT95\%PGTV $59.92 \mathrm{~Gy} / 2.14 \mathrm{~Gy} / 28 \mathrm{~F}$ and $95 \% \mathrm{PTV} 50.4$ Gy/1.8 Gy/28 F. Re-examination revealed the abnormal left supraclavicular lymph node is larger than the former. Then He received 6 cycles of chemotherapy (paclitaxel liposome $210 \mathrm{mg}$ day 1 and nedaplatin $50 \mathrm{mg}$ day $1,60 \mathrm{mg}$ day 2, q3w) from January 2020 to May 2020. During this period, the patient returned to the hospital in time according to the treatment course and did regular reexamination according to the doctor's advice. Regular re-examination every two or three months from June 2019 when showed stable disease and normal finger oxygen, but the clubbing has not been relieved. Now the patient remains in a stable condition. During concurrent radiotherapy and chemotherapy, the patient had a degree I radiation dermatitis which included skin redness, swelling and desquamation in the radiotherapy area, mild radiation esophagitis which included dysphagia and slight swallowing pain, and other adverse reactions also appeared in the chemotherapy alone such as poor appetite, nausea, and fatigue, but the blood routine, blood biochemistry, and all kinds of vital signs are all in the normal range. For the above adverse reactions, we have not made medical treatment but comfort the patient, according to CTCAE 5.0. All procedures performed in studies involving human participants were in accordance with the ethical standards of the institutional and/or national research committee(s) and with the Helsinki Declaration (as revised in 2013). Written informed consent was obtained from the patient.

\section{Discussion}

Pierre Marie and Eugen von Bamberger first described secondary HOA in 1890 and 1891, which often associated with pulmonary diseases like lung carcinoma, cystic fibrosis, or pulmonary tuberculosis (2). HPOA in malignancy, the most common is lung carcinoma (2). The association of esophageal sarcomatoid carcinoma and HPOA is extremely rare: no medical case report found in a PUBMED search 

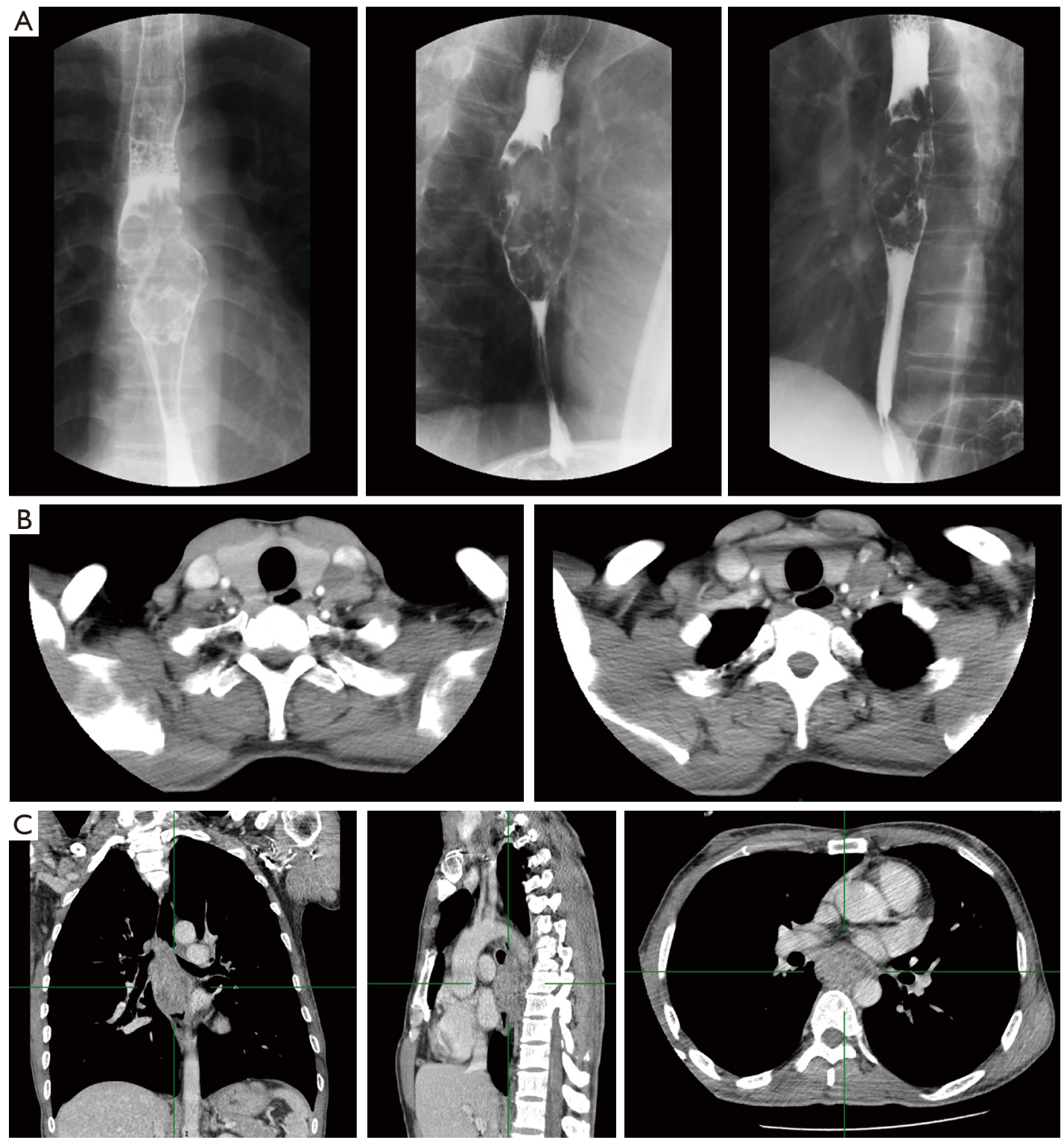

Figure 3 Masses and enlarged lymph nodes. (A) In the esophagogram, the mucosa is damaged about $7.7 \mathrm{~cm}$ in the middle thoracic esophagus, and irregular niches were seen. (B) CT scans of the trunk. A solid mass in the root of the lower neck. (C) CT scans of the trunk. A large mass $\left(3.8 \times 2.4 \times 6.6 \mathrm{~cm}^{3}\right)$ extends from the esophagus pressing the root of the pulmonary vein and invading the pericardium and diffusely involving surrounding tissues.

in the indexed English-language literature, although there have been some reports about HPOA and other pathologic types of esophageal carcinoma in the past 20 years (5-10) (Table 1). In these reports, all esophageal carcinoma patients with HPOA were male and $66.7 \%$ of them were non-epithelial esophageal carcinoma. Although there is no statistical analysis report on the relationship between esophageal carcinoma and HPOA, esophageal non- epithelial carcinoma seems more likely to cause HPOA according to the table. In this case, the primary tumor was in the middle thoracic esophagus, causing HPOA, for the following reasons. On the one hand, a constriction induced by the tumor in pulmonary vein contributed to the decrease of pulmonary artery blood flow, causing pulmonary capillary oxygen reduction, on the other hand, the intrathoracic fixation mass itself interfered respiration 

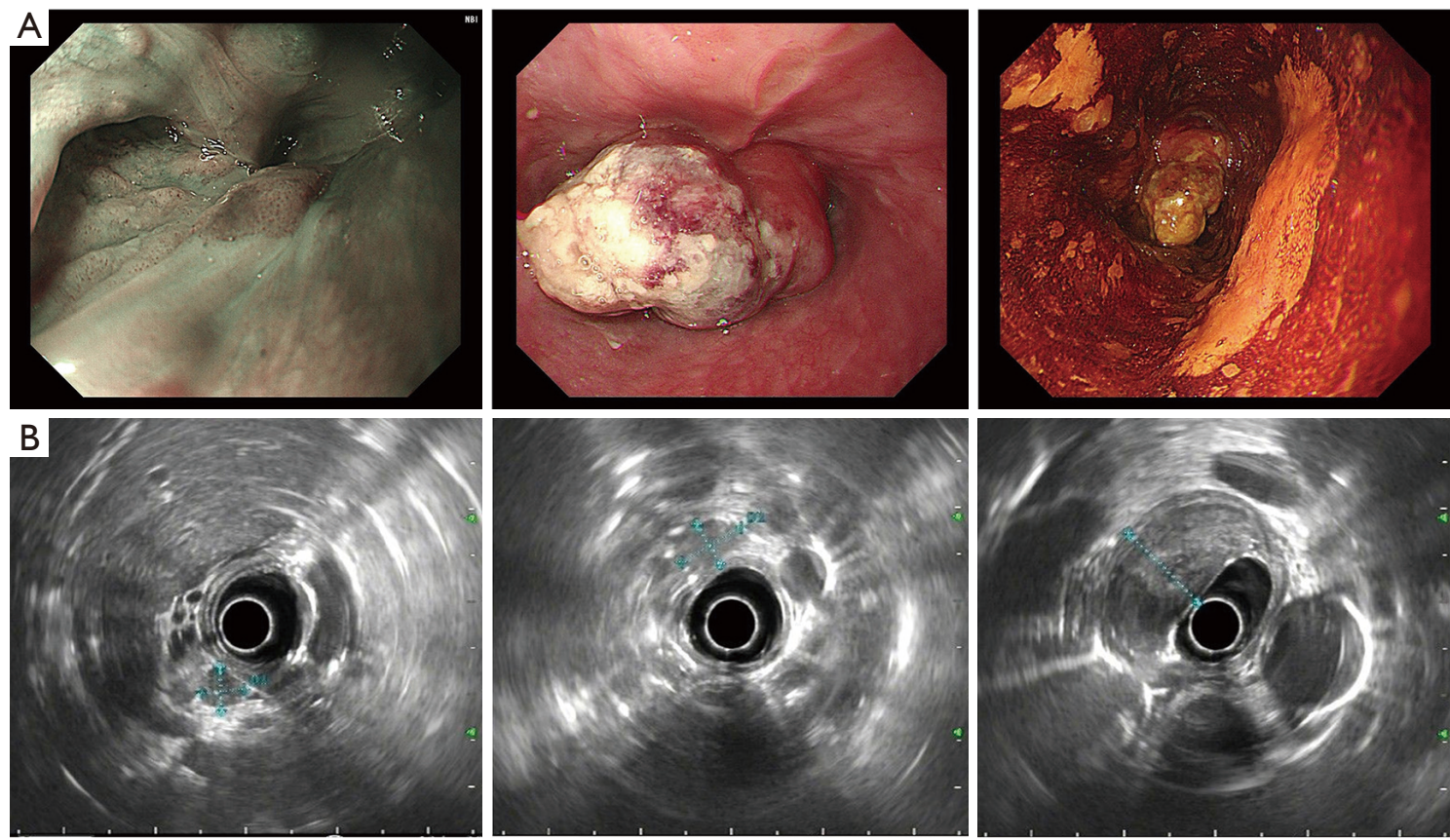

Figure 4 Endoscopic findings of the esophageal mass and hypopharyngeal abnormality. (A) Endoscopy shows that the mucosa of the left pyriform fossa is congested and rough, and a protuberant mass is in the esophagus about $27-36 \mathrm{~cm}$ from the incisor. The surface of the mass is broken and eroded, covered with a large amount of white moss. The mass is fragile and easy to bleed. The esophageal lumen with abnormal stenosis in the mass is difficult to pass through by endoscopy, but it still can do. The remaining esophageal mucosa was rough and obvious and shows a piebald lesion in iodine staining. (B) Endoscopic ultrasonography shows that the lesion is mainly located in the intrinsic muscle layer of the esophageal wall and penetrates the outer membrane of the esophageal wall with a close relationship with the surrounding tissue and unclear boundary. Abnormally enlarged lymph nodes are in the tracheoesophageal groove and beside the cardia.
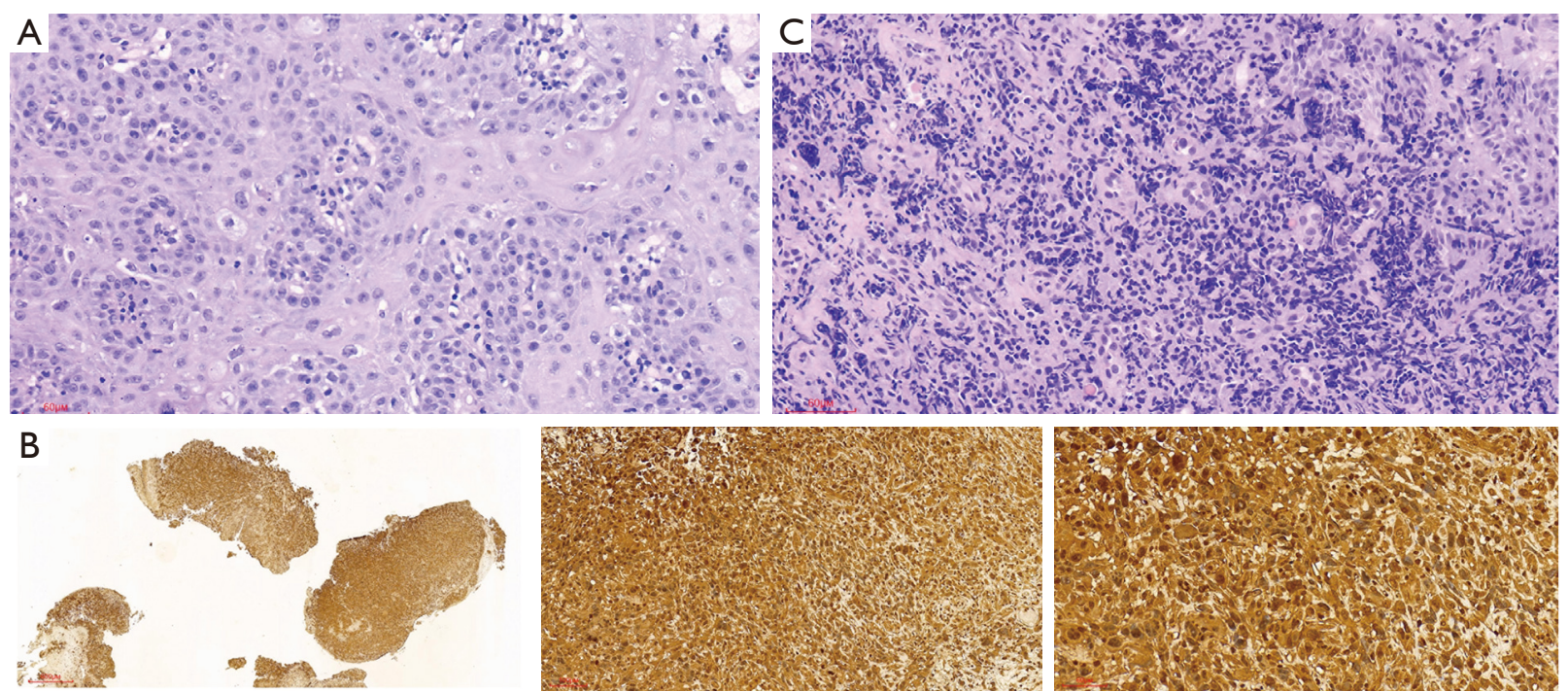

Figure 5 Pathological examination. Histopathological findings of the tumor. HE staining (A) and immunostaining of Vimentin (B) shows that spindle cells. HE staining $(\mathrm{C})$ shows diffuse dark-brownish deposition at the cytoplasmof the spindle tumor cells. (A) $\times 200$. (B) left $\times 100$; middle $\times 200$; right $\times 400$. (C) $\times 200$. 
Table 1 HPOA associated with primary esophageal carcinoma

\begin{tabular}{|c|c|c|c|c|c|c|c|}
\hline Citation & Year of report & $\begin{array}{c}\text { Numbers of } \\
\text { cases }\end{array}$ & Sex & Age (years) & Pathologic type & $\begin{array}{l}\text { Pulmonary } \\
\text { metastasis }\end{array}$ & Treatment \\
\hline Yamamoto Y et al. (5) & 2017 & 1 & M & 68 & GIST & None & $\mathrm{C}$ \\
\hline Murosaki T et al. (7) & 2015 & 1 & M & 58 & ESCC & None & - \\
\hline Morita M et al. (10) & 2003 & 1 & M & 65 & ESCC & None & $\mathrm{S}+\mathrm{CRT}$ \\
\hline
\end{tabular}

M, male; GIST, gastrointestinal tumor; NECs, neuroendocrine carcinomas; ESCC, esophageal squamous cell carcinoma; EAC, esophageal adenocarcinoma; S, surgical operation; neo, neoadjuvent; CRT, chemoradiotherapy; C, chemotherapy; RT, radiotherapy.

for increasing respiratory muscle load and occupying part of the intrapleural volume (11). Furthermore, tumor invading pericardium resulted in increased myocardial movement load and decreased ejection fraction (12). These adverse effects on the lung and heart obtained by the tumor indicated the insufficiency of blood and oxygen supply in the four limbs, manifesting clubbing fingers (toes) (2). Sarcomatoid carcinoma of epithelial origin, also called spindle cell carcinoma, with both epithelial (carcinomatous) and stromal (sarcomatoid) components, showed a biphasic appearance on histology. These spindle-shaped cells located in an undifferentiated matrix with high malignant potential, are commonly arranged in heliciform or fasciculus (4). The rare pathologic type with an approximate incidence of $0.3-2 \%$ in esophageal malignancy $(4,13)$, has been reported in some articles or case concentrated on pathological research (4,13-15). All the reported patients were without HPOA.

Compared with esophageal cancer, HPOA is more common in lung cancer (16). HPOA incidence in cases of primary lung carcinoma in the USA published in the 1980 s put the occurrence at $0.8 \%$ (17). In recent years, Cruz et al. have reported a male Afro-descendant 57-year-old patient was bronchial adenocarcinoma with HPOA and abnormal antinuclear antibodies and anti$\mathrm{Sm}$ antibodies (18). The clinical symptoms of HPOA are caused by tumors, which are easily misdiagnosed as autoimmune diseases. We should pay attention to this point in the diagnosis and treatment in all kinds of thoracic cancer combined with HPOA.

The rate of locoregional metastasis was $33-65 \%$ and the tumor doubling time was $2.2-5$ months in esophageal sarcomatoid carcinoma $(13,14,19)$. Regional lymph nodes followed by lungs and pleura were commonly involved. It preferentially metastasized to the lymphatics in a rapid and aggressive manner but showed a better prognosis than esophageal squamous cell carcinoma. The 5 -year survival in Stage III/IV was $16.2 \%$ and radical resection with lymphadenectomy was the first treatment way for operable patients with esophageal sarcomatoid carcinoma (13). Our patient had no opportunity of surgery after surgical evaluation so that chemoradiotherapy with a conventional division of radical dose is the most appropriate first-time treatment for him in view of KPS 80 and no fundamental disease.

The patient's condition has been effectively controlled by chemotherapy and radiotherapy, but the five-year survival rate of patients with advanced esophageal carcinoma is still very low under the existing traditional treatment methods, and the adverse reactions also contribute to low life quality. We look forward to the breakthrough of immunotherapy and molecular targeting therapy to improve the situation.

Although the patient had lost the opportunity of operation when he went to see a doctor, after concurrent chemoradiotherapy and multicycle chemotherapy, the patient is now in a stable condition with KPS 70. Now, he is particularly satisfied with the treatments. As the mainstay of the family, although he can no longer bear the heavy work, he can at least spend an uneventful but precious time with his family and accompany his child to grow up.

Based on our report, although HPOA associated with primary esophageal sarcomatoid carcinoma is a rare disease, it should not be underestimated. 


\section{Acknowledgments}

We thank the patient and all the authors in the work. Funding: This report is supported by National key research and development program (2017YFC1311000).

\section{Footnote}

Reporting Checklist: The authors have completed the CARE reporting checklist. Available at http://dx.doi.org/10.21037/ apm-20-1309

Conflicts of Interest: All authors have completed the ICMJE uniform disclosure form (available at http://dx.doi. org/10.21037/apm-20-1309). The authors have no conflicts of interest to declare.

Ethical Statement: The authors are accountable for all aspects of the work in ensuring that questions related to the accuracy or integrity of any part of the work are appropriately investigated and resolved. All procedures performed in studies involving human participants were in accordance with the ethical standards of the institutional and/or national research committee(s) and with the Helsinki Declaration (as revised in 2013). Written informed consent was obtained from the patient.

Open Access Statement: This is an Open Access article distributed in accordance with the Creative Commons Attribution-NonCommercial-NoDerivs 4.0 International License (CC BY-NC-ND 4.0), which permits the noncommercial replication and distribution of the article with the strict proviso that no changes or edits are made and the original work is properly cited (including links to both the formal publication through the relevant DOI and the license). See: https://creativecommons.org/licenses/by-nc-nd/4.0/.

\section{References}

1. Krugh M, Vaidya PN. Osteoarthropathy Hypertrophic. StatPearls. Treasure Island (FL); 2020.

2. Chakraborty RK, Sharma S. Secondary Hypertrophic Osteoarthropathy. StatPearls. Treasure Island (FL); 2020.

3. Wu CC, Chen CJ. Esophageal carcinoma. $\mathrm{N}$ Engl J Med 2015;372:1472.

4. Raza MA, Mazzara PF. Sarcomatoid carcinoma of esophagus. Arch Pathol Lab Med 2011;135:945-8.

5. Yamamoto Y, Sasaki Y, Kougame M, et al. Giant oesophageal gastrointestinal stromal tumour presenting with dyspnoea and clubbed fingers. BMJ Case Rep 2017;2017:bcr2017220540.

6. Saif MW, Vethody C. Poorly Differentiated Neuroendocrine Tumor of the Esophagus with Hypertrophic Osteoarthropathy and Brain Metastasis: A Success Story. Cureus 2016;8:e646.

7. Murosaki T, Mori K, Nagashima T, et al. Hypertrophic osteoarthropathy associated with esophageal cancer. Intern Med 2015;54:357-8.

8. Wechalekar MD, Kennedy NA, Ahern M, et al. Esophageal adenocarcinoma and hypertrophic osteoarthropathy with improvement following resection of esophageal cancer. J Clin Rheumatol 2011;17:323-4.

9. Axel J, Weickert U, Dancygier H. Gastrointestinal tumor (GIST) of the esophagus in a 34-year-old man: clubbed fingers and alopecia arealis as an early paraneoplastic phenomenon. Dtsch Med Wochenschr 2005;130:2380-3.

10. Morita M, Sakaguchi Y, Kuma S, et al. Hypertrophic osteoarthropathy associated with esophageal cancer. Ann Thorac Surg 2003;76:1744-6.

11. Tucker WD, Mahajan K. Anatomy, Blood Vessels. StatPearls. Treasure Island (FL); 2020.

12. Janicki JS. Influence of the pericardium and ventricular interdependence on left ventricular diastolic and systolic function in patients with heart failure. Circulation 1990;81:III15-20.

13. Zhang B, Xiao Q, Yang D, et al. Spindle cell carcinoma of the esophagus: A multicenter analysis in comparison with typical squamous cell carcinoma. Medicine (Baltimore) 2016;95:e4768.

14. Handra-Luca A, Terris B, Couvelard A, et al. Spindle cell squamous carcinoma of the oesophagus: an analysis of 17 cases, with new immunohistochemical evidence for a clonal origin. Histopathology 2001;39:125-32.

15. Pateria P, Muwanwella N, Chai M, et al. Synchronous oesophageal and gastric sarcomatoid carcinoma: first reported case in Australia. BMJ Case Rep 2018;2018:bcr2018225092.

16. Martinez-Lavin M, Mansilla J, Pineda C, et al. Evidence of hypertrophic osteoarthropathy in human skeletal remains from pre-Hispanic Mesoamerica. Ann Intern Med 1994;120:238-41.

17. Segal AM, Mackenzie AH. Hypertrophic osteoarthropathy: a 10-year retrospective analysis. Semin Arthritis Rheum 1982;12:220-32. 
18. Cruz C, Rocha M, Andrade D, et al. Hypertrophic pulmonary osteoarthropathy with positive antinuclear antibodies: case report. Case Rep Oncol 2012;5:308-12.

Cite this article as: Zhao M, Du Y, Peng Q, Hui Z. Hypertrophic pulmonary osteoarthropathy with esophageal sarcomatoid carcinoma: a case report. Ann Palliat Med 2021;10(5):5862-5869. doi: 10.21037/apm-20-1309
19. Sasajima K, Taniguchi Y, Morino K, et al. Rapid growth of a pseudosarcoma of the esophagus. J Clin Gastroenterol 1988;10:533-6. 